\title{
A NOTE ABOUT COMPLEXITY OF LENS SPACES
}

\author{
Maria Rita CASALI - Paola CRISTOFORI
}

September 7, 2021

\begin{abstract}
Within crystallization theory, (Matveev's) complexity of a 3-manifold can be estimated by means of the combinatorial notion of GM-complexity. In this paper, we prove that the GM-complexity of any lens space $L(p, q)$, with $p \geq 3$, is bounded by $S(p, q)-3$, where $S(p, q)$ denotes the sum of all partial quotients in the expansion of $\frac{q}{p}$ as a regular continued fraction. The above upper bound had been already established with regard to complexity; its sharpness was conjectured by Matveev himself and has been recently proved for some infinite families of lens spaces by Jaco, Rubinstein and Tillmann. As a consequence, infinite classes of 3-manifolds turn out to exist, where complexity and GM-complexity coincide.

Moreover, we present and briefly analyze results arising from crystallization catalogues up to order 32 , which prompt us to conjecture, for any lens space $L(p, q)$ with $p \geq 3$, the following relation: $k(L(p, q))=5+2 c(L(p, q))$, where $c(M)$ denotes the complexity of a 3 -manifold $M$ and $k(M)+1$ is half the minimum order of a crystallization of $M$.
\end{abstract}

Keywords: 3-manifold, complexity, crystallization, 2-bridge knot, 4-plat, lens space

Mathematics Subject Classification (2010): 57Q15, 57M27, 57M25, 57M15.

\section{Introduction}

The complexity $c(M)$ of a 3-manifold $M$ was originally defined by Matveev as the minimum number of true vertices among all almost simple spines of $M([24])[1$

\footnotetext{
${ }^{1}$ Recall that a spine of $M$ is a 2-dimensional subpolyhedron $P$ of $\operatorname{Int}(M)$, such that $M$ (or $M$ minus an open 3-ball, if $M$ is closed) collapses to $P$; moreover, a spine $P$ is almost simple if the link of each point of $\mathrm{P}$ can be embedded in the 1-skeleton of a 3 -simplex. A point whose link is the whole 1-skeleton of the 3 -simplex is called a true vertex of $P$.
} 
The 3 -sphere, the real projective space, the lens space $L(3,1)$ and the spherical bundles $\mathbb{S}^{1} \times \mathbb{S}^{2}$ and $\mathbb{S}^{1} \tilde{\times} \mathbb{S}^{2}$ have complexity zero. Excluding these examples, it is well-known that the complexity of a closed prime 3-manifold $M$ coincides with the number of tetrahedra in a minimal pseudocomplex triangulating $M$. Therefore, it is possible to obtain tables of 3-manifolds for increasing values of their complexity, simply by generating all triangulations (resp. spines) with a given number of tetrahedra (resp. true vertices) and by identifying the related manifolds. Up-to-date censuses of closed orientable (resp. non-orientable) 3manifolds are available at the Web page http://www.matlas.math.csu.ru/ (resp. in [6, Appendix]) 2

The complexity of a manifold is generally hard to compute from the theoretical point of view, leaving aside the concrete enumeration of its spines.

As far as lens spaces are concerned, a well-known estimation for complexity exists: $c(L(p, q)) \leq S(p, q)-3$, where $S(p, q)$ denotes the sum of all partial quotients in the expansion of $\frac{q}{p}$ as a regular continued fraction ([25] and [21]). The sharpness of this estimation was conjectured by Matveev himself ([25, page 77]); in the last decade Jaco, Rubinstein and Tillmann succeeded to prove that it is sharp for some infinite families of lens spaces, comprehending $L(2 r, 1)$ and $L(4 r, 2 r-1), \forall r \geq 2$ (see [21] and [22]).

In the present paper we approach the problem of computing complexity of lens spaces by making use of crystallization theory, i.e. by a combinatorial tool to represent any PLmanifold via suitable edge-coloured graphs, called crystallizations: see [19], [1] and [2] for a survey on the topic. Within this theory, the invariant GM-complexity has been introduced for 3-manifolds: see [8] (resp. [9] and [11]) for the closed non-orientable (resp. orientable) case, and [13] for the extension to 3-manifolds with non-empty boundary.

The GM-complexity of a 3-manifold $M$ (denoted by $c_{G M}(M)$ ) turns out to be an upper bound for the complexity of $M$ : via GM-complexity, direct estimations of complexity have been obtained for interesting classes of 3-manifolds, both in the closed case (see [9] for 2 -fold and 3-fold branched coverings of $\mathbb{S}^{3}$, and for 3-manifolds obtained by Dehn surgery on framed links in $\mathbb{S}^{3}$ ) and in the boundary case (see [13] for torus knot complements).

Conjectures about significant upper bounds for infinite families of 3-manifolds have also arisen by the analysis of existing crystallization catalogues, which have been generated up to 30 vertices ([7], [12, 3]).

In section 3, we present results concerning the extension of the above catalogues to 32 vertices, with regard to the orientable case: as a consequence, the conjectures are proved up to this order. In particular, we remark that $c_{G M}(L(p, q))=c(L(p, q))=S(p, q)-3$ for any lens space involved in crystallization catalogues.

On the other hand, the main result of the present paper states that $S(p, q)-3$ is an upper bound for the GM-complexity of any lens space $L(p, q)$ with $p \geq 3$ (Proposition 6(a)).

The coincidence with the known upper bound for $c(L(p, q))$ emphasizes the strong relationship between GM-complexity and complexity, which is suggested by the analysis of the crystallization catalogues (see for example [14, Conjecture 7], where equality between the

\footnotetext{
${ }^{2}$ See the references in [2, section 1] for subsequent results about the classification of 3-manifolds according to complexity, established by various authors.
} 
two invariants is conjectured for all closed 3-manifolds).

In particular, lens spaces of type $L(2 r, 1)$ and $L(4 r, 2 r-1), \forall r \geq 2$, turn out to be infinite classes of 3-manifolds where complexity and GM-complexity actually coincide (see Corollary (7).

Within crystallization theory, a slightly different complexity notion has been introduced and analyzed, too: a closed PL $n$-manifold $M^{n}$ is said to have gem-complexity $k\left(M^{n}\right)=p-1$ if $2 p$ is the minimum order of a crystallization representing $M^{n}$ (see [23], [7] and [10]).

In section 4 (Proposition 6 (b)) we prove that $k(L(p, q)) \leq 2 S(p, q)-1 \forall p \geq 2$. The sharpness of the above bound for a double infinite family of lens spaces (Corollary 9) and for all lens spaces up to complexity 5 suggests the equality to hold in the general case (Conjecture 8).

Moreover, we prove that, whenever $c(L(p, q))=S(p, q)-3$, then $k(L(p, q)) \leq 5+$ $2 c(L(p, q))$ follows (Proposition [11). Actually, in Proposition [10, we list some infinite families of lens spaces for which equality holds. Therefore, it is natural to conjecture it for all lens spaces, i.e. $k(L(p, q))=5+2 c(L(p, q)) \quad \forall p \geq 3$ (Conjecture 12).

\section{Crystallizations, gem-complexity and GM-complexity}

Edge-coloured graphs are a representation tool for the whole class of piecewise linear (PL) manifolds, without restrictions about dimension, connectedness, orientability or boundary properties. In the present work, however, we will deal only with closed and connected PL-manifolds of dimension $n=3$; hence, we will briefly review basic notions and results of the theory with respect to this particular case.

A 4-coloured graph (without boundary) is a pair $(\Gamma, \gamma)$, where $\Gamma=(V(\Gamma), E(\Gamma))$ is a regular multigraph (i.e. it may include multiple edges, but no loop) of degree four and $\gamma: E(\Gamma) \rightarrow \Delta_{3}=\{0,1,2,3\}$ is a proper edge-coloration (i.e. it is injective when restricted to the set of edges incident to any vertex of $\Gamma$ ).

The elements of the set $\Delta_{3}$ are called the colours of $\Gamma$; thus, for every $i \in \Delta_{3}$, an $i$ coloured edge is an element $e \in E(\Gamma)$ such that $\gamma(e)=i$. For every $i, j \in \Delta_{3}$ let $\Gamma_{\hat{\imath}}$ (resp. $\Gamma_{i j}$ ) (resp. $\left.\Gamma_{\hat{\imath} \hat{\jmath}}\right)$ be the subgraph obtained from $(\Gamma, \gamma)$ by deleting all the edges of colour $i$ (resp. $c \in \Delta_{3}-\{i, j\}$ ) (resp. $c \in\{i, j\}$ ). The connected components of $\Gamma_{i j}\left(\right.$ resp. $\Gamma_{\hat{\imath}}$ ) (resp. $\Gamma_{\hat{\imath} \hat{\jmath}}$ ) are called $\{i, j\}$-coloured cycles (resp. $\hat{\imath}$-residues) (resp. $\{\hat{\imath}, \hat{\jmath}\}$-coloured cycles) of $\Gamma$, and their number is denoted by $g_{i j}$ (resp. $g_{\hat{\imath}}$ ) (resp. $g_{\hat{\imath} \hat{\jmath}}$ ). A 4-coloured graph $(\Gamma, \gamma)$ is called contracted iff, for each $i \in \Delta_{3}$, the subgraph $\Gamma_{\hat{\imath}}$ is connected (i.e. iff $g_{\hat{\imath}}=1 \forall i \in \Delta_{3}$ ).

Every 4-coloured graph $(\Gamma, \gamma)$ may be thought of as the combinatorial visualization of a 3-dimensional labelled pseudocomplex $K(\Gamma)$, which is constructed according to the following instructions:

- for each vertex $v \in V(\Gamma)$, take a 3 -simplex $\sigma(v)$, with its vertices labelled $0,1,2,3$;

- for each $j$-coloured edge between $v$ and $w(v, w \in V(\Gamma))$, identify the 2-dimensional faces of $\sigma(v)$ and $\sigma(w)$ opposite to the vertex labelled $j$, so that equally labelled vertices coincide. 
In case $K(\Gamma)$ triangulates a (closed) PL 3-manifold $M$, then $(\Gamma, \gamma)$ is called a gem (gem $=$ graph encoded manifold) representing $M 3$

Finally, a 4-coloured graph representing a (closed) 3-manifold $M$ is a crystallization of $M$ if it is also a contracted graph; by construction, it is not difficult to check that this is equivalent to requiring that the associated pseudocomplex $K(\Gamma)$ contains exactly one $i$-labelled vertex, for every $i \in \Delta_{3}$. The representation theory of PL-manifolds by edgecoloured graphs is often called crystallization theory, since it has been proved that every PLmanifold admits a crystallization: see Pezzana Theorem and its subsequent improvements ([19] or [1]).

A cellular embedding of a coloured graph into a surface is said to be regular if its regions are bounded by the images of bicoloured cycles; interesting results of crystallization theory (mainly related to an $n$-dimensional extension of Heegaard genus, called regular genus and introduced in [20]) rely on the existence of this type of embeddings for graphs representing manifolds of arbitrary dimension.

As far as the 3-dimensional case is concerned, it is well-known that, if $(\Gamma, \gamma)$ is a crystallization of an orientable (resp. non-orientable) manifold $M$, then for every pair $\alpha, \beta \in \Delta_{3}$, there exists a regular embedding $i_{\alpha, \beta}: \Gamma \rightarrow F_{\alpha \beta}$, such that $F_{\alpha \beta}$ is the closed orientable (resp. non-orientable) surface of genus $g_{\alpha \beta}-1$. The minimum genus of $F_{\alpha \beta}$ taken over all pairs $\alpha, \beta \in \Delta_{3}$ is called the regular genus of $(\Gamma, \gamma)$.

Let now $\mathcal{D}$ (resp. $\left.\mathcal{D}^{\prime}\right)$ be an arbitrarily chosen $\{\alpha, \beta\}$-coloured (resp. $\{\hat{\alpha}, \hat{\beta}\}$-coloured) cycle of a crystallization $(\Gamma, \gamma)$; we denote by $\mathcal{R}_{\mathcal{D}, \mathcal{D}^{\prime}}$ the set of regions of $F_{\alpha \beta}-i_{\alpha, \beta}\left(\left(\Gamma_{\alpha \beta}-\right.\right.$ $\left.\mathcal{D}) \cup\left(\Gamma_{\hat{\alpha} \hat{\beta}}-\mathcal{D}^{\prime}\right)\right)$.

Definition 1. Let $M$ be a closed 3-manifold, and let $(\Gamma, \gamma)$ be a crystallization of $M$. With the above notations, the Gem-Matveev complexity (or GM-complexity, for short) of $\Gamma$ is defined as the non-negative integer

$$
c_{G M}(\Gamma)=\min \left\{\# V(\Gamma)-\#\left(V(\mathcal{D}) \cup V\left(\mathcal{D}^{\prime}\right) \cup V(\Xi)\right) / \mathcal{D} \in \Gamma_{\alpha \beta}, \mathcal{D}^{\prime} \in \Gamma_{\hat{\alpha} \hat{\beta}}, \Xi \in \mathcal{R}_{\mathcal{D}, \mathcal{D}^{\prime}}\right\},
$$

while the Gem-Matveev complexity (or GM-complexity, for short) of $M$ is defined as

$$
c_{G M}(M)=\min \left\{c_{G M}(\Gamma) /(\Gamma, \gamma) \text { crystallization of } M\right\}
$$

Remark 1 The notion of GM-complexity can also be extended to non-contracted gems (see [9, Definition 4]); however, it has been proved that, for each closed 3-manifold $M$, the minimum value of GM-complexity is always realized by crystallizations ([14, Proposition 6]). For this reason, in this paper we restrict our attention to GM-complexity of crystallizations.

The following key result, due to [8], justifies the choice of terminology:

\footnotetext{
${ }^{3}$ The construction of $K(\Gamma)$ directly ensures that, if $(\Gamma, \gamma)$ is a gem of $M$, then $M$ is orientable (resp. non-orientable) iff $\Gamma$ is bipartite (resp. non-bipartite).
} 
Proposition 1 For every closed 3-manifold M, GM-complexity gives an upper bound for complexity of $M$ :

$$
c(M) \leq c_{G M}(M)
$$

Within crystallization theory, another quite natural notion of complexity has been introduced $([23],[7])$ :

Definition 2. For each closed PL $n$-manifold $M^{n}$, gem-complexity is defined as the nonnegative integer

$$
k\left(M^{n}\right)=\min \left\{\frac{\# V(\Gamma)}{2}-1 /(\Gamma, \gamma) \text { crystallization of } M^{n}\right\}
$$

As far as dimension three is concerned, existing 3-manifold censuses via crystallizations (see paragraph 3) induce to investigate possible relations between complexity $c(M)$ and gem-complexity $k(M)$. In particular, in [11], the following conjecture is stated:

Conjecture $2 \quad k(M) \leq 5+2 c(M)$ for any closed orientable 3-manifold $M$.

\section{Up-to-date results from crystallization catalogues}

The totally combinatorial nature of graphs encoding manifolds, makes the theory particularly effective for generating catalogues of PL-manifolds for increasing order of the representing graphs (i.e. for increasing values of $k\left(M^{n}\right)$ ). The main tool for the algorithmic generation of tables of crystallizations is the code, a numerical "string" which completely describes the combinatorial structure of a coloured graph, up to colour-isomorphisms (see [15]); afterwards, suitable moves on gems (see [18]) are applied, to yield a classification procedure which allows to detect crystallizations of the same manifold.

In particular, the generation and classification procedures have been successfully developed in dimension 3 , both in the orientable and non-orientable case, allowing the complete topological identification of each involved 3-manifold: see [23] and [12] (resp. [3]) for censuses of orientable (resp. non-orientable) 3-manifolds up to gem-complexity 14 .

In the orientable case (to which we restrict our attention in the present paper), the obtained results allow to state:

Proposition 3 There are exactly 110 closed prime orientable 3-manifolds, up to gemcomplexity 14. 
Details about the quoted catalogues are available at the Web page: http://cdm.unimo.it/home/matematica/casali.mariarita/CATALOGUES.htm.

In particular, Table 1, Table 2 and Table 3 of the above WEB page contain the JSJdecomposition of each manifold up to gem-complexity 14 and an analysis of the distribution of these manifolds with respect to complexity and geometry.

By optimizing the program code and by exploiting high-powered computers, we succeeded in extending the above crystallization catalogue to 3-manifolds with $k(M)=15.5$

The classification of the involved manifolds has been performed by means of the powerful computer program "3-Manifold Recognizer"6

The analysis of the catalogue yields the following result, concerning the geometries of the involved manifolds and the comparison between $k(M)$ and $c(M)$ :

Proposition 4 There are exactly 110 closed prime orientable 3-manifolds with gemcomplexity 15. They are:

- 38 elliptic 3-manifolds (in particular: 20 lens spaces, which are exactly those with complexity five; 18 other elliptic, of which 11 with complexity six 7 and seven with complexity seven);

- four Nil Seifert 3-manifolds (one with complexity seven, one with complexity eight and two with complexity nine);

- ten 3-manifolds with Sol geometry (one with complexity seven 8 , three with complexity eight and six with complexity nine);

- two 3-manifolds with $\mathbb{H}^{2} \times \mathbb{R}$ geometry (which are exactly those with complexity eight);

- 34 3-manifolds with $\widetilde{S L}_{2}(\mathbb{R})$ geometry (six with complexity seven, 19 with complexity eight and nine with complexity nine);

- 17 non-geometric 3-manifolds (five with complexity eight and 12 with complexity nine);

- five hyperbolic 3-manifolds (one with complexity nine, three with complexity ten and one with complexity 11) 9

\footnotetext{
${ }^{4}$ In particular, we made use of CINECA facilities, such as a IBM SP Power6 system for high-performance computing, which are available in virtue of some established Italian Supercomputing Resource Allocation (ISCRA) projects.

${ }^{5}$ The same task has been independently achieved by Tarkaev and Fominykh, too: see [25, page 367].

6 "3-Manifold Recognizer" has been written by V. Tarkaev as an application of the results about recognition of 3-manifolds obtained by $\mathrm{S}$. Matveev and his research group. It is available on the Web: http://www.matlas.math.csu.ru/

${ }^{7}$ They are exactly the missing ones with that complexity in the crystallization catalogue for $k \leq 14$.

${ }^{8}$ It is exactly the missing one with that complexity in the crystallization catalogue for $k \leq 14$.

${ }^{9}$ They are all Dehn fillings of the complement of $6_{1}^{3}$ (i.e. the chain link with three components in $\mathbb{S}^{3}$ ).
} 
Apart from $\mathbb{S}^{3}, L(2,1), L(3,1)$ and the sphere bundle $\mathbb{S}^{2} \times \mathbb{S}^{1}$ (i.e. the ones with complexity zero), the distribution of prime 3 -manifolds with $k(M) \leq 15$ with respect to complexity and geometry is summarized in the following table (where the symbol $x / n$ means that $x 3$-manifolds appear in the catalogue among the $n$ ones having the corresponding complexity and geometry, and bold character is used to indicate the presence of all manifolds of the considered type).

\begin{tabular}{|c|c|c|c|c|c|c|c|c|c|c|c|}
\hline complexity & 1 & 2 & 3 & 4 & 5 & 6 & 7 & 8 & 9 & 10 & 11 \\
\hline lens & $2 / 2$ & $3 / 3$ & $6 / 6$ & $10 / 10$ & $20 / 20$ & $0 / 36$ & $0 / 72$ & $0 / 136$ & $0 / 272$ & $0 / 528$ & $0 / 1056$ \\
\hline other elliptic & - & $1 / 1$ & $1 / 1$ & $4 / 4$ & $11 / 11$ & $25 / 25$ & $7 / 45$ & $0 / 78$ & $0 / 142$ & $0 / 270$ & $0 / 526$ \\
\hline $\mathbb{E}^{3}$ & - & - & - & - & - & $6 / 6$ & - & - & - & - & - \\
\hline$\sim$ Nil & - & - & - & - & - & $7 / 7$ & $4 / 10$ & $3 / 14$ & $2 / 15$ & $0 / 15$ & $0 / 15$ \\
\hline$\widetilde{S L}_{2}(\mathbb{R})$ & - & - & - & - & - & - & $19 / 39$ & $24 / 162$ & $11 / 513$ & $0 / 1416$ & $0 / 3696$ \\
\hline Sol & - & - & - & - & - & - & $5 / 5$ & $5 / 9$ & $6 / 23$ & 0/39 & $0 / 83$ \\
\hline non-geometric & - & - & - & - & - & - & $4 / 4$ & $6 / 35$ & $14 / 185$ & $0 / 777$ & $0 / 2921$ \\
\hline $\mathbb{H}^{2} \times \mathbb{R}$ & - & - & - & - & - & - & - & $2 / 2$ & - & $0 / 8$ & $0 / 4$ \\
\hline hyperbolic & - & - & - & - & - & - & - & - & $3 / 4$ & $4 / 25$ & $1 / 120$ \\
\hline TOTAL & $2 / 2$ & $4 / 4$ & $7 / 7$ & $14 / 14$ & $31 / 31$ & $38 / 74$ & $39 / 175$ & $40 / 436$ & $36 / 1154$ & $4 / 3078$ & $1 / 8421$ \\
\hline
\end{tabular}

Prime 3-manifolds with complexity $c \neq 0$ and gem-complexity $k \leq 15$.

It is worthwhile to note that this new table emphasizes an idea already suggested by the cases up to gem-complexity 14: for any fixed $c$, subsequent crystallization catalogues, for increasing order, appear to cover first the most "complicated" types of complexity $c$ 3-manifolds and then the simplest ones, such as lens spaces (see the above quoted Table 3).

On the other hand 3-manifold censuses via crystallizations suggest that "restricted" gemcomplexity implies "restricted" complexity, and viceversa: see [7, Section 5], [8, Remark 1] and [11, Propositions 5 and 6]). In particular, if we improve the known results in the orientable case with the ones obtained from the analysis of the catalogue of order 32 bipartite crystallizations, we can state 10

Proposition 5 Let $M$ be a closed orientable prime 3-manifold.

(a) If $c(M) \leq 6$ and $M \neq L(p, q)$, then $k(M)<5+2 c(M)$;

(b) if $M=L(p, q)$ and $1 \leq c(M) \leq 5$, then $k(M)=5+2 c(M)$.

\section{Results about lens spaces}

We are now able to state our main result:

Proposition 6 (Main Theorem) Let $L(p, q)$ be a lens space. Then:

(a) $c_{G M}(L(p, q)) \leq S(p, q)-3 \quad \forall p \geq 3$;

\footnotetext{
${ }^{10}$ Note that the results of Proposition 5 strengthen Conjecture 2.
} 
(b) $k(L(p, q)) \leq 2 \cdot S(p, q)-1 \quad \forall p \geq 2$.

Moreover, if $c(L(p, q)) \leq 5$, then:

$$
k(L(p, q))=2 \cdot S(p, q)-1 \quad \text { and } \quad c_{G M}(L(p, q))=c(L(p, q))=S(p, q)-3 .
$$

Proof. Without loss of generality, we may assume $p, q \in \mathbb{Z}$ coprime with $1 \leq q \leq \frac{p}{2}$. Let $\mathfrak{b}(p, q)$ be the 2-bridge knot or link, with numbers $p$ and $q$ in Schubert's normal form, so that $L(p, q)$ is the 2 -fold covering of $\mathbb{S}^{3}$ branched over $\mathfrak{b}(p, q)$ (see [5, Proposition 12.3]).

According to [5, Proposition 12.13], $\mathfrak{b}(p, q)$ admits a presentation $\bar{P}$ as a 4-plat with a defining braid $\mathfrak{z}=\sigma_{2}^{a_{1}} \cdot \sigma_{1}^{-a_{2}} \ldots \sigma_{1}^{-a_{m-1}} \cdot \sigma_{2}^{a_{m}}$, with $a_{i}>0 \forall i \in\{1, \ldots, m\}$ and $m$ odd, where the $a_{i}$ 's are the quotients of the continued fraction $\left[a_{1}, \ldots, a_{m}\right]=\frac{q}{p}$. In Fig. 1 the presentation $\bar{P}$ is shown, for the case $p=21, q=8$ : in fact, $\frac{8}{21}=[2,1,1,1,2]$. Note that $\bar{P}$ admits $\sum_{i=1}^{m} a_{i}=S(p, q)$ crossings; for sake of notational simplicity, we set $S(p, q)=\bar{s}$.

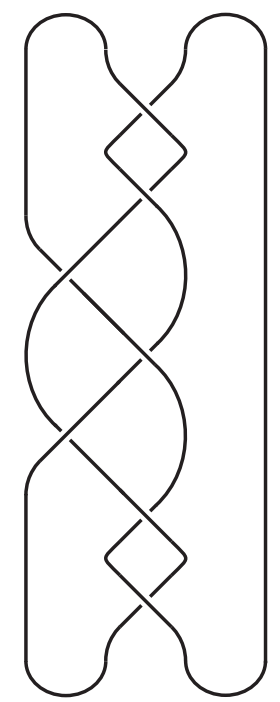

Figure 1: a 4-plat presentation of $\mathfrak{b}(21,8)$

Let now $(\bar{\Gamma}, \bar{\gamma})=F(\bar{P})$ be the crystallization obtained by means of Ferri's construction of crystallizations of 2-fold branched coverings (see [17]), applied to $\bar{P}$ considered as a bridge presentation of $\mathfrak{b}(p, q)$, with a bridge for each crossing point 11

$(\bar{\Gamma}, \bar{\gamma})$ may be easily "drawn over" $\bar{P}$, as it can be seen in Fig. 2 for the case $p=21, q=8$, starting from the bridge presentation of the 4-plat presentation of $\mathfrak{b}(21,8)$ depicted in Fig. 1.

In this way, each $\{0,1\}$-coloured cycle of $\bar{\Gamma}$ arises from a bridge and has length four; hence, $(\bar{\Gamma}, \bar{\gamma})$ turns out to have $4 \bar{s}$ vertices, which directly proves part (b) of the statement.

\footnotetext{
${ }^{11}$ Crystallizations arising from Ferri's construction are said to be 2-symmetric (see [16]) since an involution exists on their edges, which exchanges colour 0 (resp. 2) with colour 1 (resp. 3): this involution can be thought of as the planar symmetry whose axis contains all the bridges of the given knot or link presentation.
} 

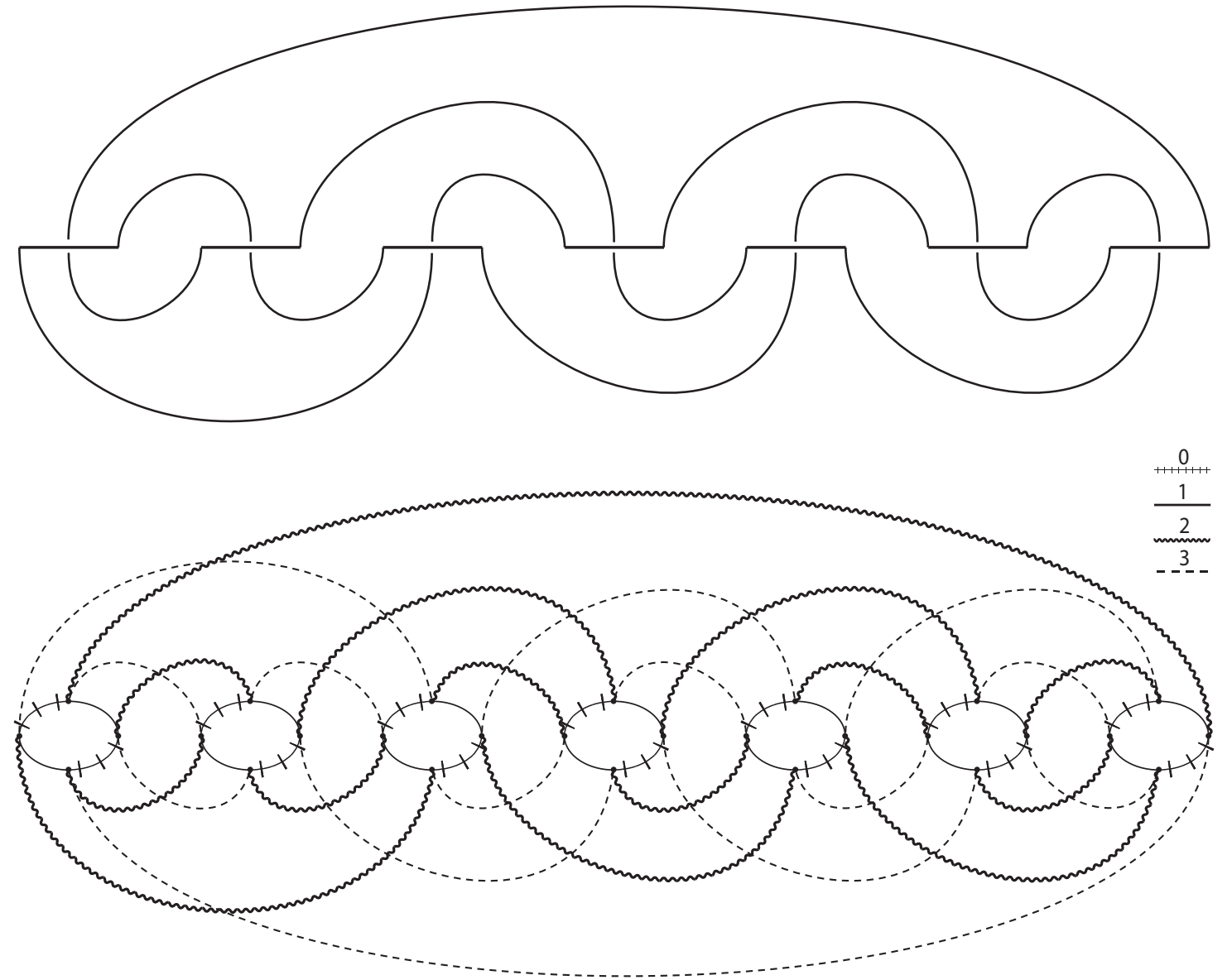

Figure 2: a bridge presentation of $\mathfrak{b}(21,8)$ and the associated crystallization of $L(21,8)$

Suppose now $p \geq 3$, i.e. $\bar{s} \geq 3$. Let us label the crossings of $\bar{P}$ according to the braid sequence $\sigma_{2}^{a_{1}} \cdot \sigma_{1}^{-a_{2}} \ldots \sigma_{1}^{-a_{m-1}} \cdot \sigma_{2}^{a_{m}}$ and let $\pi$ be the "ideal" axis containing all bridges of $\bar{P}$ with the orientation induced by the sequence $a_{1}, \ldots, a_{m}$. For each crossing $c_{j}(1 \leq j \leq \bar{s})$, we label $v_{j, 1}, v_{j, 2}, v_{j, 3}, v_{j, 4}$ the vertices of its associated $\{0,1\}$-coloured cycle, so that $v_{j, 2}$ and $v_{j, 4}$ belong to $\pi$ and the sequence $v_{1,2}, v_{1,4}, v_{2,2}, v_{2,4}, \ldots, v_{\bar{s}, 2}, v_{\bar{s}, 4}$ is consistent with the fixed orientation of $\pi$. Moreover, $v_{j, 1}, v_{j, 2}$ and $v_{j, 3}, v_{j, 4}$ (resp. $v_{j, 2}, v_{j, 3}$ and $v_{j, 4}, v_{j, 1}$ ) are 0 -adjacent (resp. 1-adjacent), for each $j \in 1, \ldots, \bar{s}$. The 2-coloured edges follow the arcs of $\bar{P}$, while 3 -coloured edges are obtained from arcs of $\bar{P}$ via symmetry with respect to the axis $\pi$ (see again Fig. 2).

As a consequence we have that, for each $j, k \in\{1, \ldots, \bar{s}\}$, vertices $v_{j, 2}$ and $v_{k, 1}$ (resp. $v_{j, 3}$ and $\left.v_{k, 4}\right)$ are 3 -adjacent iff $v_{j, 2}$ and $v_{k, 3}$ (resp. $v_{j, 1}$ and $v_{k, 4}$ ) are 2-adjacent.

In order to estimate $c_{G M}(\bar{\Gamma})$ (via Definition 1), let us now fix $\{\alpha, \beta\}=\{0,2\}$ and $\left\{\alpha^{\prime}, \beta^{\prime}\right\}=\{1,3\}$, and let us consider the $\{0,2\}$-coloured (resp. $\{1,3\}$-coloured) cycle $\mathcal{D}$ (resp. $\mathcal{D}^{\prime}$ ) corresponding to the boundary of the "external" region $\bar{R}$ of $\bar{P}$ (resp. obtained via symmetry from the boundary of the "external" region $\bar{R}$ of $\bar{P}$ ), and the region $\Xi \in \mathcal{R}_{\mathcal{D}, \mathcal{D}^{\prime}}$ induced by the inner region $\bar{R}^{\prime}$ of $\bar{P}$ containing the fourth string of the 4-braid associated to the 4-plat $\bar{P}$.

Let us define the following sets of integers: 


$$
\begin{gathered}
I_{1}=\left\{j \in\{2, \ldots, \bar{s}-1\} \mid c_{j} \text { corresponds to a factor } \sigma_{1} \text { in } \mathfrak{z}\right\} \cup\{\bar{s}\} \\
I_{2}=\left\{j \in\{1, \ldots, \bar{s}-1\} \mid c_{j} \text { corresponds to a factor } \sigma_{2} \text { in } \mathfrak{z}\right\} .
\end{gathered}
$$

Since the boundary of $\bar{R}$ contains the crossing points $c_{j}$ with $j \in I_{1} \cup\{1\}$, it is not difficult to check that $\mathcal{D} \cup \mathcal{D}^{\prime}$ consists of the vertices $\left\{v_{j, i} / j \in I_{1}, i=1,3,4\right\} \cup\left\{v_{1,1}, v_{1,2}, v_{1,3}\right\}$.

On the other hand, the structure of $\bar{P}$ and the symmetry properties of $\bar{\Gamma}$, which arise from Ferri's construction, allow to check that the region $\Xi$ can be obtained by the union of the four regions bounded by the following cycles of $\bar{\Gamma}$ :

- the $\{1,2\}$-coloured cycle corresponding to $\bar{R}^{\prime}$; its vertex-set is $\left\{v_{j, i} \mid j \in I_{2}, i=\right.$ $1,4\} \cup\left\{v_{\bar{s}, 1}, v_{\bar{s}, 4}\right\}$,

- the $\{0,3\}$-coloured cycle obtained via symmetry from $\bar{R}^{\prime}$; its vertex-set is $\left\{v_{j, i} \mid j \in\right.$ $\left.I_{2}, i=3,4\right\} \cup\left\{v_{\bar{s}, 3}, v_{\bar{s}, 4}\right\}$,

- the $\{0,1\}$-coloured cycle corresponding to the last crossing $c_{\bar{s}}$,

- the $\{2,3\}$-coloured cycle containing the edge corresponding to the fourth string of the braid $\mathfrak{z}$ (whose vertices are exactly $\left\{v_{1,1}, v_{1,3}, v_{2,2}, v_{\bar{s}, 4}\right\}$, since $q<\frac{p}{2}$ implies $a_{1} \geq 2$ ).

Hence, $V(\mathcal{D}) \cup V\left(\mathcal{D}^{\prime}\right) \cup V(\Xi)$ contains all vertices $v_{j, i}$ with $1 \leq j \leq \bar{s}, \quad i \in\{1,3,4\}$ and the vertices $v_{j, 2}$ with $j \in\{1,2, \bar{s}\}$. As a consequence,

$$
V(\bar{\Gamma})-\left(V(\mathcal{D}) \cup V\left(\mathcal{D}^{\prime}\right) \cup V(\Xi)\right)=\left\{v_{j, 2} / 3 \leq j \leq \bar{s}-1\right\},
$$

which implies $c_{G M}(\bar{\Gamma}) \leq \bar{s}-3$. So, part (a) of the statement directly follows.

Finally, the last part of the statement can be checked by means of the existing crystallization catalogues, up to order 32 (presented in the previous paragraph).

Proposition 6 (a), together with the results of [21] and [22], directly proves the existence of infinite classes of 3-manifolds where complexity and GM-complexity coincide:

\section{Corollary 7}

- $c_{G M}(L(2 r, 1))=c(L(2 r, 1))=2 r-3$, for each $r \geq 2$;

- $c_{G M}(L(4 r, 2 r-1))=c(L(4 r, 2 r-1))=r$, for each $r \geq 2$;

- $c_{G M}(L((r+2)(t+1)+1, t+1))=c(L((r+2)(t+1)+1, t+1))=r+t$, for each $t>r>1, r$ odd and $t$ even;

- $c_{G M}(L((r+1)(t+2)+1, t+2))=c(L((r+1)(t+2)+1, t+2))=r+t$, for each $t>r>1, r$ even and $t$ odd. 
With regard to gem-complexity, Proposition 6(b) suggests the following conjecture:

Conjecture $8 k(L(p, q))=2 \cdot S(p, q)-1 \quad \forall p \geq 2$.

Actually, a recent result by Swartz $([26]) 12$ proves that, for each $q, r \geq 1$ odd, $k(L(q r+$ $1, q))=2(r+q)-1$; since $S(q r+1, q)=q+r$ trivially holds $\forall r, q$, we can state:

Corollary 9 Conjecture 8 is true for any lens space $L(q r+1, q)$, with $r, q \geq 1$ odd.

Remark 2 Note that our Main Theorem yields $k(L(p, 1)) \leq 2 p-1$. Actually, in this case, the described crystallization $(\bar{\Gamma}, \bar{\gamma})$ - which, by [26], realizes gem-complexity of $L(p, 1)$ in the case of $p$ even - is the well-known standard crystallization of $L(p, 1)$, having $4 p$ vertices and regular genus one. On the contrary, if $q \neq 1$ is assumed, the standard crystallization of $L(p, q)$ has regular genus one and $4 p$ vertices, too, but it does not realize minimum order, since it contains clusters or, more generally, structures that can be eliminated (see [12, section 6] or [18, section 5] for details).

Remark 3 Proposition 6(b) yields a general result, which admits [4, Theorem 2.6(v)] and [4, Theorem 2.6(vi)] as particular cases. Note that, in the cited paper, the authors' approach via fundamental groups allows to rediscover examples of minimal crystallizations already presented in [23] (see http://cdm.unimo.it/home/matematica/casali.mariarita/Table1.pdf).

Finally, we can summarize the relation between complexity and gem-complexity of lens spaces as follows:

\section{Proposition 10}

(a) Let $L(p, q)$ be a lens space, with $p \geq 3$. Then

$$
k(L(p, q))=5+2 \cdot c(L(p, q))
$$

whenever:

$\left(a_{1}\right) c(L(p, q)) \leq 5$

$\left(a_{2}\right) p$ is even and $q=1$;

$\left(a_{3}\right) p=(r+2)(t+1)+1$ and $q=t+1$, for $t>r>1, r$ odd and $t$ even;

$\left(a_{4}\right) p=(r+1)(t+2)+1$ and $q=t+2$, for $t>r>1, r$ even and $t$ odd.

\footnotetext{
${ }^{12}$ The preprint [26] was posted in ArXiv (http://arxiv.org/abs/1310.1991v1) a few days after we posted the first version of the present paper (http://arxiv.org/abs/1309.5728v1). It makes use of our Proposition 6(b) in order to prove that $4(q+r)$ is exactly the minimum order of a crystallization of $L(q r+1, q), \forall r, q \geq 1$ odd.
} 
(b) For each $r, q \geq 1$ odd, then

$$
k(L(q r+1, q)) \geq 5+2 \cdot c(L(q r+1, q)) ;
$$

(c) for each $r \geq 2$, then

$$
k(L(4 r, 2 r-1)) \leq 5+2 \cdot c(L(4 r, 2 r-1)) .
$$

Proof. Part $\left(a_{1}\right)$, already stated in Proposition [6(a), directly follows from the analysis of crystallization catalogues up to gem-complexity 15.

In order to prove part $\left(a_{2}\right)$ (resp. $\left.\left(a_{3}\right)\right)$ (resp. $\left.\left(a_{4}\right)\right)$, the equality $k(L(q r+1, q))=$ $2(r+q)-1$ (which holds $\forall r, q \geq 1$ odd: see Corollary 9) has to be applied to the involved infinite class of lens spaces, together with [21, Theorem 1] (resp. [21, Theorem 2]) (resp. [21, Theorem 3]).

Part (b) follows from the equality $k(L(q r+1, q)=2(r+q)-1, \forall r, q \geq 1$ odd, too, by making use of the well-known general estimation $c(L(p, q)) \leq S(p, q)-3$.

Part (c) is a direct consequence of Proposition 6(b) and of [22, Corollary 3].

Moreover, we can state

Proposition 11 Assuming Matveev's conjecture $c(L(p, q))=S(p, q)-3$ to be true, then

$$
k(L(p, q)) \leq 5+2 \cdot c(L(p, q)) \quad \forall p \geq 2 .
$$

In particular, $k(L(p, q)) \leq 5+2 \cdot c(L(p, q))$ for each lens space $L(p, q)$ with $6 \leq$ $c(L(p, q)) \leq 12$ and equality holds if $p=q r+1$, with $q, r$ odd.

Proof. If $c(L(p, q))=S(p, q)-3$ is assumed $\forall p \geq 3$, then $k(L(p, q)) \leq 2 \cdot S(p, q)-1=$ $5+2 \cdot c(L(p, q))$ trivially follows from part (b) of our Main Theorem.

In case $6 \leq c(L(p, q)) \leq 12$, condition $c(L(p, q))=S(p, q)-3$ is always satisfied (see http://www.matlas.math.csu.ru/) and, therefore, $k(L(p, q)) \leq 5+2 \cdot c(L(p, q))$. On the other hand, if, further, $p=q r+1$ ( $q, r$ odd) then part (b) of Proposition 10 applies to ensure equality.

The above Propositions 10 and 11 naturally suggest the following:

Conjecture $12 k(L(p, q))=5+2 \cdot c(L(p, q)) \quad \forall p \geq 3$.

Remark 4 A slightly different notion of GM-complexity for closed 3-manifolds was originally defined by taking into account only the values of $c_{G M}(\Gamma)$, when $(\Gamma, \gamma)$ is a minimal crystallization with respect to the number of vertices: see [8, Definition 3]. As regards lens spaces, existing crystallization catalogues prove that this restricted notion actually coincides with the one including all crystallizations. Moreover, the arguments of the present paper induce to conjecture - according to the above Conjectures 8 and 12 - that complexity of any lens space is always realized by GM-complexity of a minimal crystallization (despite what happens in the general case: see [11, Proposition 7 and Remark 4]). 
Acknowledgements. We wish to thank E. Fominykh for the helpful discussions which originated this paper. We are also indebted to him, to S. Matveev and V. Tarkaev for the use of "3-Manifold Recognizer".

This work was performed under the auspices of the G.N.S.A.G.A. of I.N.d.A.M. (Italy) and financially supported by M.I.U.R. of Italy, University of Modena and Reggio Emilia, funds for selected research topics.

\section{References}

[1] P. Bandieri - M.R. Casali - C. Gagliardi, Representing manifolds by crystallization theory: foundations, improvements and related results, Atti Sem. Mat. Fis. Univ. Modena Suppl. 49 (2001), 283-337.

[2] P. Bandieri, M.R. Casali, P. Cristofori, L. Grasselli and M. Mulazzani, Computational aspects of crystallization theory: complexity, catalogues and classification of 3manifolds, Atti Sem. Mat. Fis. Univ. Modena 58 (2011), 11-45.

[3] P. Bandieri - P. Cristofori - C. Gagliardi, Nonorientable 3-manifolds admitting coloured triangulations with at most 30 tetrahedra, J. Knot Theory Ramifications 18 (2009), 381-395.

[4] B. Basak - B. Datta, Minimal crystallizations of 3-manifolds, arXiv:1308.6137 [math.GT], 2013.

[5] G. Burde - H. Zieschang, Knots, Walter de Gruyter, Berlin - New York, 1985.

[6] B.A. Burton, Enumeration of non-orientable 3-manifolds using face-paring graphs and union-find, Discrete Comput. Geom. 38 (2007), 527-571.

[7] M.R. Casali, Classification of non-orientable 3-manifolds admitting decompositions into $\leq 26$ coloured tetrahedra, Acta Appl. Math. 54 (1999), 75-97.

[8] M.R. Casali, Computing Matveev's complexity of non-orientable 3-manifolds via crystallization theory, Topology Appl. 144 (2004), 201-209.

[9] M.R. Casali, Estimating Matveev's complexity via crystallization theory, Discrete Math. 307 (2007), 704-714.

[10] M. R. Casali, Catalogues of PL-manifolds and complexity estimations via crystallization theory, Oberwolfach Reports - Workshop "TRIANGULATIONS" (April 29th - May 04th 2012), 24 (2012), 58-61. [DOI: 10.4171/OWR/2012/24]

[11] M.R. Casali - P. Cristofori, Computing Matveev's complexity via crystallization theory: the orientable case, Acta Appl. Math. 92 (2006), 113-123.

[12] M.R. Casali - P. Cristofori, A catalogue of orientable 3-manifolds triangulated by 30 coloured tetrahedra, J. Knot Theory Ramifications 17 (2008), 1-23. 
[13] M.R. Casali - P. Cristofori, Computing Matveev's complexity via crystallization theory: the boundary case, J. Knot Theory Ramifications 22 (8) (2013), 1350038 (30 pages). [DOI: $10.1142 / \mathrm{S} 0218216513500387]$

[14] M.R. Casali - P. Cristofori - M. Mulazzani, Complexity computation for compact 3manifolds via crystallizations and Heegaard diagrams, Topology Appl. 159 (13) (2012), 3042-3048. [DOI: 10.1016/j.topol.2012.05.016]

[15] M.R. Casali - C. Gagliardi, A code for m-bipartite edge-coloured graphs, Rend. Ist. Mat. Univ. Trieste 32 (2001) 55-76.

[16] M.R. Casali - L. Grasselli, 2-symmetric crystallizations and 2-fold branched covering of $\mathbb{S}^{3}$, Discrete Math. 87 (1991), 9-22.

[17] M. Ferri, Crystallisations of 2-fold branched coverings of $\mathbb{S}^{3}$, Proc. Amer. Math. Soc. 73 (1979), 271-276.

[18] M. Ferri - C. Gagliardi, Crystallization moves, Pacific J. Math. 100 (1982), 85-103.

[19] M. Ferri - C. Gagliardi - L. Grasselli, A graph-theoretical representation of PLmanifolds. A survey on crystallizations, Aequationes Math. 31 (1986), 121-141.

[20] C. Gagliardi, Extending the concept of genus to dimension n, Proc. Amer. Math. Soc. 81 (1981), 473-481.

[21] W. Jaco - H. Rubinstein - S. Tillmann, Minimal triangulations for an infinite family of lens spaces, J. Topol. 2 (2009), 157-180.

[22] W. Jaco - H. Rubinstein - S. Tillmann, Coverings and Minimal Triangulations of 3Manifolds, Algebr. Geom. Topol. 11 (2011), 1257-1265.

[23] S. Lins, Gems, computers and attractors for 3-manifolds, Knots and Everything 5, World Scientific, 1995.

[24] S. Matveev, Complexity theory of three-dimensional manifolds, Acta Appl. Math. 19 (1990), 101-130.

[25] S. Matveev, Algorithmic Topology and Classification of 3-manifolds, Algorithms and Computation in Mathematics 9, Springer-Verlag, 2007.

[26] E. Swartz, The average dual surface of a cohomology class and minimal simplicial decompositions of infinitely many lens spaces, arXiv:1310.1991 [math.GT], 2013. 